\title{
Observations sur les nids de deux chalicodomes et leurs occupants en Sardaigne (Italie)
}

\section{Par Nicolas J Vereecken *, Pietro NIOLU **, Eric DUFRÊNE *** et Gérard LEGofF ****}

\begin{abstract}
We here provide field observations made in spring 2008 in Sardinia on the nesting biology of two mason bees, Megachile (Chalicodoma) parietina (GEOFFroy in FOURCROY 1785) and M. (C.) sicula (Rossi 1792) whose females occasionally construct egg-shaped nests perched on tree twigs. Active nests of $M$. (C.) parietina were also exploited by females of Osmia signata ERICHSON 1835 and those of M. (C.) sicula were found to be parasitised by Leucospis gigas FABRICIUS 1793.
\end{abstract}

Résumé. Nous fournissons ici quelques observations de terrain réalisées au printemps 2008 en Sardaigne et relatives à la nidification de deux espèces de chalicodomes, Megachile (Chalicodoma) parietina (GEOFFROY dans FOURCROY 1785) et $M$. (C.) sicula (Rossi 1792) qui construisent parfois des nids ovoïdes perchés sur des rameaux d'arbres. Les nids de $M$. (C.) parietina étaient également exploités par des femelles d'Osmia signata ERICHSON 1835 et ceux de $M$. (C.) sicula se sont avérés être parasités par Leucospis gigas FABRICIUS 1793.

Mots-clés. Megachile, Chalicodoma, nidification, parasitisme.

\section{Introduction}

Les chalicodomes (Megachile (Chalicodoma) spp., Megachilidae) sont des abeilles sauvages solitaires et maçonnes qui ont fait l'objet d'observations depuis l'époque de Réaumur et d'études détaillées sur leur nidification et leur orientation par Fabre (1882) dans ses Souvenirs Entomologiques. Deux spectaculaires espèces de chalicodomes (14-17mm) peuvent encore être rencontrées dans des milieux préservés en Méditerranée : le chalicodome des murailles, Megachile (Chalicodoma) parietina (GEOFFrOY dans FOURCROY 1785) et le chalicodome de Sicile, $M$. (C.) sicula (ROSSI 1792 ), plus méridional que la première espèce et qui, contrairement à ce qu'indique son épithète spécifique, n'est pas spécifique à la Sicile : elle se rencontre aussi en France (Tkalcu 1977; Rasmont et al. 1995), dans la Péninsule Ibérique (Ceballos 1956), en Italie (Pagliano 1993), en Grèce, dans les îles de Méditerranée et en Afrique du Nord (Schmiedeknecht 1930).

\section{Observations en Sardaigne}

Au cours de prospections réalisées dans le nord de la Sardaigne (Italie) fin avril 2008 dans les environs d'Alghero, nous avons eu le chance de parcourir des milieux abondamment fleuris qui abritaient une importante diversité entomologique. Nous détaillons cidessous quelques observations relatives à ces deux espèces de chalicodomes, leurs nids et leurs insectes associés.

\footnotetext{
* Nicolas J Vereecken, Evolution Biologique \& Ecologie, Université Libre de Bruxelles, av. FD Roosevelt 50 CP 160/12, B-1050 Bruxelles, Belgique. E-mail: nicolas.vereecken@ulb.ac.be

** Pietro Niolu, Via Sassari 130, SS-07041 Alghero, Sardaigne, Italie. *** Eric Dufrêne, Ecologie Systématique \& Evolution (ESE, UMR8079, CNRS \& Univ. Paris-Sud Orsay, AgroParisTech), Université Paris-Sud XI, Bât. 362, F-91405 Orsay Cedex, France.

E-mail: eric.dufrene@u-psud.fr

**** Gérard Le Goff, 44 rue Albert Malet, F-76360 Barentin, France.
}

Les chalicodomes sont des abeilles de gros gabarit, cetains individus pouvant atteindre $18 \mathrm{~mm}$. Les femelles du chalicodome des murailles sont d'une livrée totalement noire et leurs ailes sont largement enfumées et aux reflets violacés (Figure 1A). Les mâles de cette espèce (voir photo de couverture de ce numéro) présentent une pilosité brun-ferrugineux et des métatarses le plus souvent sombres, ce qui les distingue notamment des mâles de $M$. (C.) pyrenaica (LEPELETIER 1841). Les femelles du chalicodome de Sicile présentent un habitus tout à fait caractéristique : leur thorax est relativement uniformément couvert d'une pilosité ferrugineuse et la cuticule de leurs pattes est colorée d'un rouge-orange vif diagnostique que l'on observe également chez les mâles. Les deux espèces sont actives à la même période de l'année, d'avril à juin, et il n'est pas rare de contacter les deux taxons sur les mêmes sites.

La construction du nid est une opération longue et minutieuse à laquelle s'attelle les femelles de chalicodomes pendant toute leur courte vie et ce dès que l'accouplement a eu lieu. Les femelles s'orientent rapidement vers des zones dégagées (p.ex. des sentiers) où elles récoltent les petits cailloux et la poussière qui serviront de base à la confection du mortier de leur nid (Figures 1A-B). Ces éléments sont mélangés à la salive et l'eau pour former une substance malléable qui est appliquée sur la surface plane d'un rocher bien exposé aux rayons du soleil et souvent à l'abri des intempéries. Les femelles débutent la construction du nid par l'élaboration de cellules larvaires individuelles qui seront ensuite recouvertes intégralement du même mortier pour former une structure lisse et homogène rappelant l'aspect du crépi et qui épouse le plus souvent remarquablement les formes naturelles du support choisi (Figures 1C-F). 

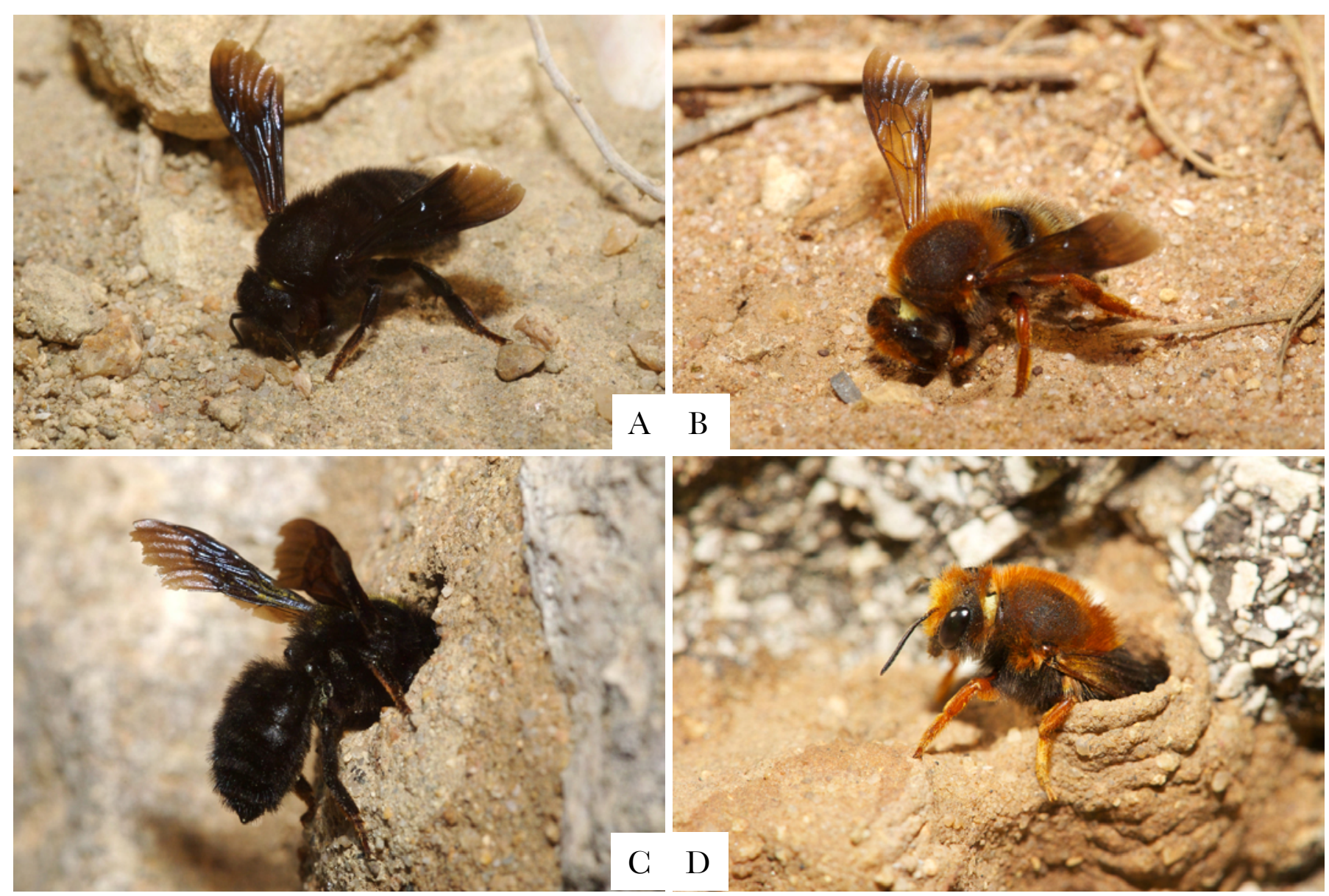

A B
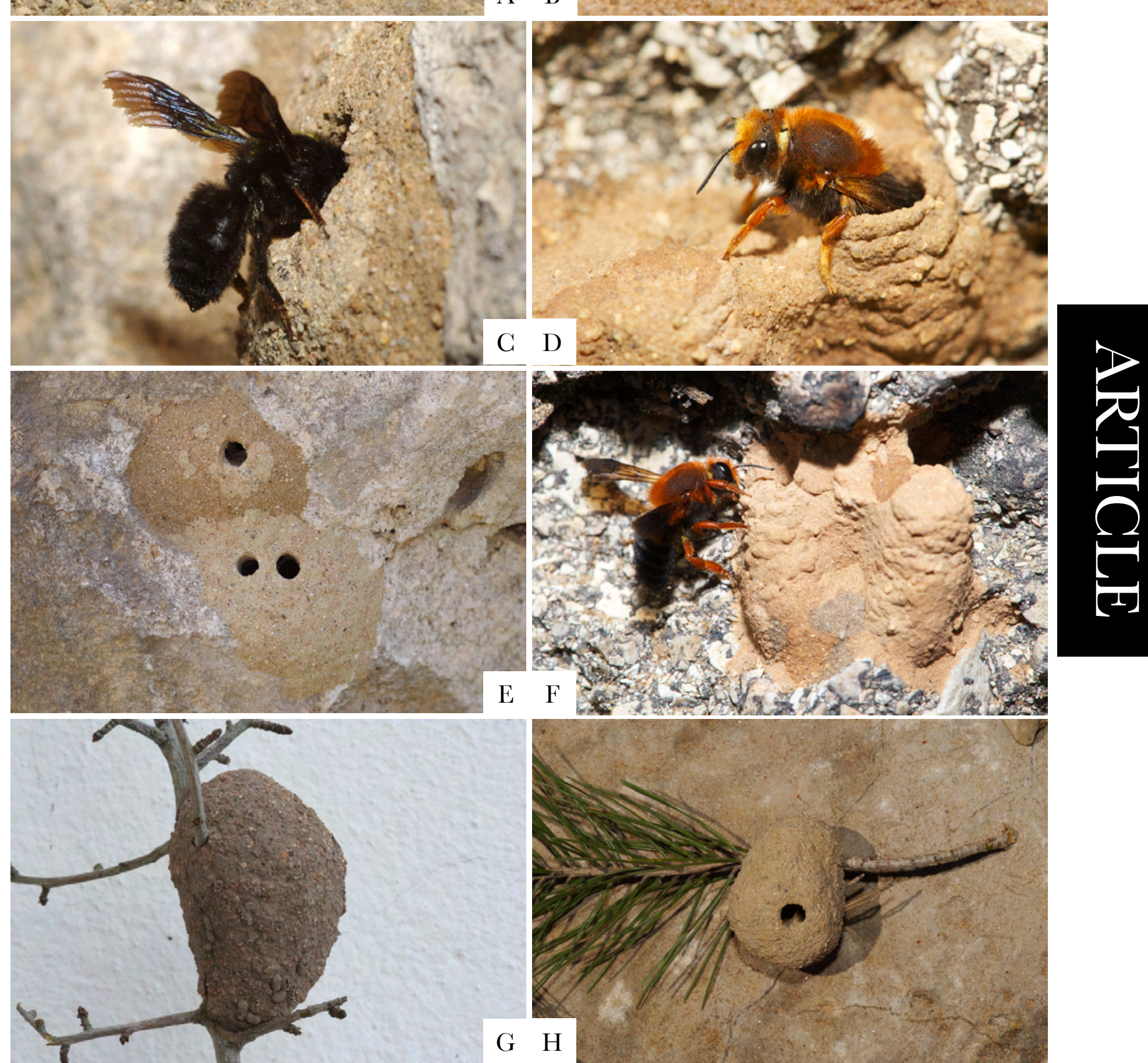

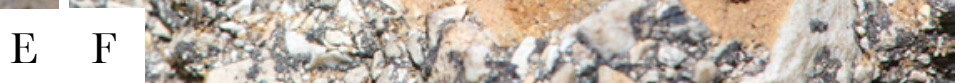

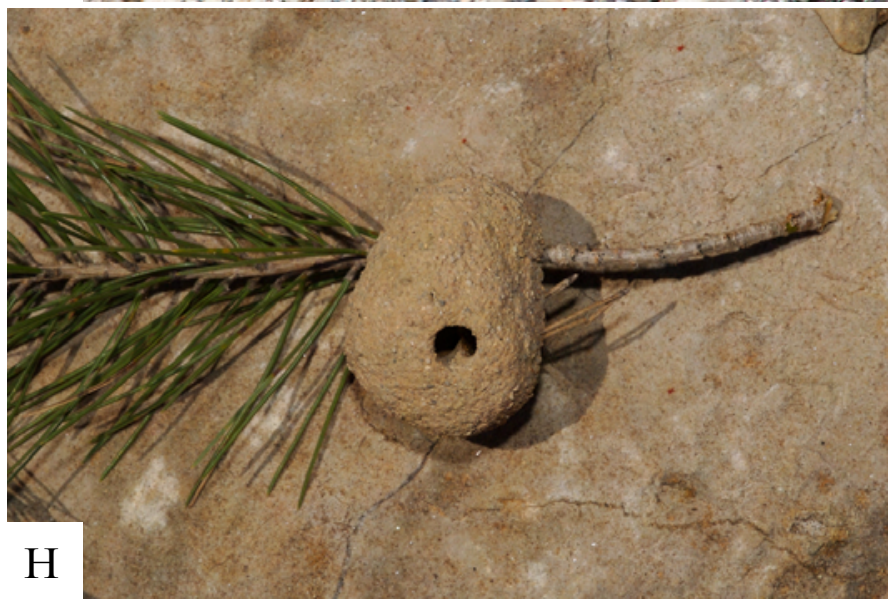

Figure 1. Ecologie de la nidification des femelles de chalicodomes, Megachile (Chalicodoma) parietina (GEOFFroY in FourCROY, 1785) et M. (C.) sicula (RossI, 1792). A-B: Les femelles récoltent des matériaux de construction sur les terrains nus et rocailleux. Le tout est aggloméré en dômes par les femelles (C-D) qui construisent plusieurs cellules sur des rochers plats, bien exposés au soleil et le plus souvent à l'abri des intempéries (E-F). A plusieurs reprises, nous avons pu observer des nids ovoïdes accrochés aux branches de certains arbres comme un nid de M. parietina sur un poirier (G) ou un nid de M. sicula sur un pin (H) (Photos NJ Vereecken sauf $\mathbf{G}$ par P Niolu) 

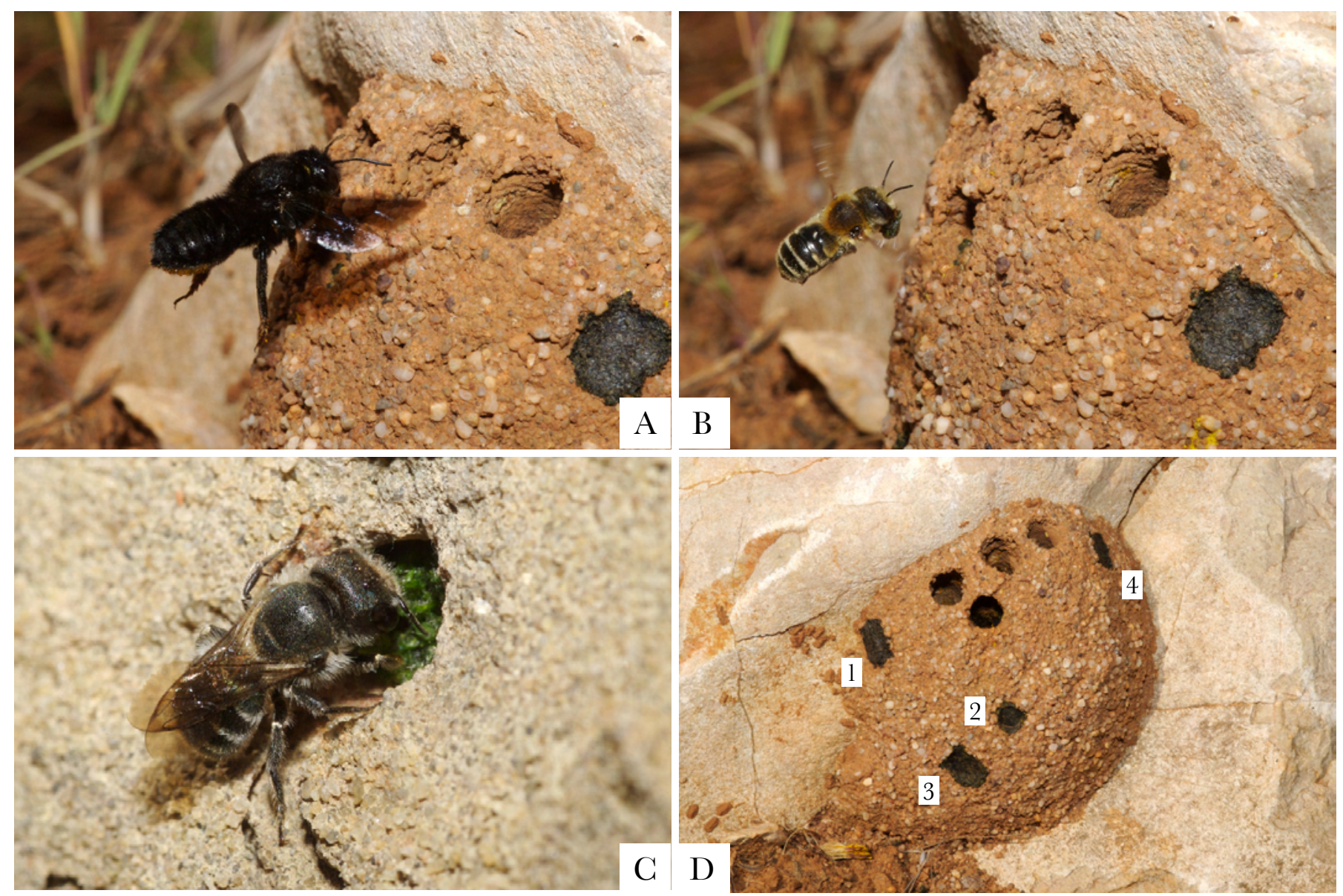

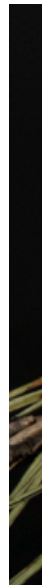
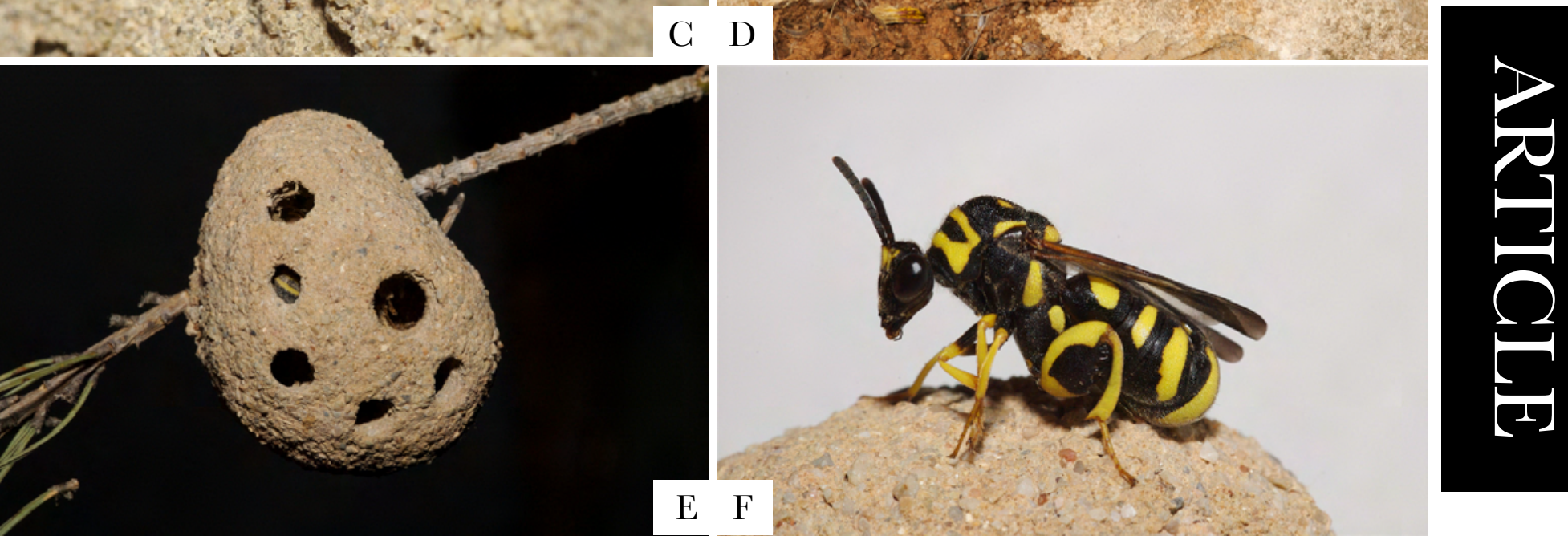

Figure 2. A-B: Observations d'un nid de M. (C.) parietina (GEOFFROY in FouRCROY, 1785) en activité et dont certaines galeries d'émergence sont exploitées par une femelle d'Osmia signata ERICHSON 1835; C: les femelles Osmia (Helicosmia) caerulescens (L. 1758) réalisent un bouchon végétal pour sceller l'entrée de leur nid après l'approvisionnement des cellules larvaires; $\mathbf{D}$ : la femelle d'O. signata avait déjà réalisé 4 cellules dans le nid de $M$. (C.) parietina; E-F: nid arboricole de M. (C.) sicula (RossI, 1792) duquel ont successivement émergé 6 femelles de la guêpe-coucou Leucospis gigas FABRICIUs 1793 (Leucospidae) (Photos NJ Vereecken).

Une fois terminés, les nids sont donc à peu près parfaitement camouflés dans leur environnement et leur localisation nécessite parfois de nombreuses heures de recherche. Nous avons trouvé plusieurs de ces nids lors de nos prospections, et nous avons également eu la chance de découvrir plusieurs nids arboricoles (Figures $1 \mathrm{G}-\mathrm{H})$ que nous pensions initialement être l'oeuvre de guêpes maçonnes. Ces nids étaient accrochés à des branches de poiriers et de pins, et nous en avons prélevé deux d'entre eux afin d'en identifier les occupants.

Tout en réalisant ces premières observations, nous nous sommes également attardés sur certains nids de M. (G.) parietina en activité dont quelques trous d'émergence semblaient scellés à l'aide d'un bouchon vraisemblablement d'origine végétale (Figure 2A). Cette technique nous a rappelé les matériaux utilisés par les osmies du sous-genre Helicosmia lors de la confection de leur nid, et en particulier le cas d'Osmia (Helicosmia) caerulescens (L. 1758) que nous côtoyons régulièrement dans la moitié nord de la France et en Belgique. Quelques heures d'affût aux heures les plus chaudes de la journée nous ont permis d'observer une "squatteuse" de ce nid identifiée comme $O$. $(H$.) signata ERICHSON 1835, une espèce connue pour exploiter cavités préexistantes et autres nids d'abeilles abandonnés (Müller 2010). La petite osmie avait déjà complété quatre cellules larvaires (Figure 2D) alors que la femelle de $M$. (C.) parietina était toujours en activité (Figure 2A) et ne semblait pas perturbée par ses allers et venues. 
L. gigas, ces derniers étant d'un gabarit proche de celui des chalicodomes. Les larves de $M$. (C.) parietina sont principalement nourries de pollen récolté par les femelles sur des inflorescences d'Onobrychis spp. (Fabaceae) (Figure 3) (Müller et al. 1997) et il a été estimé qu'une cellule larvaire de $M$. (C.) parietina contient en moyenne $100 \mathrm{~mm}^{3}$ de pollen, ce qui nécessite la visite de 1139 fleurs par une femelle de chalicodome, soit presque deux fois plus que les ressources accumulées par les femelles de Melitta dimidiata MORAWITz 1876 (Hym. Melittidae) (Figure 3), une autre espèce d'abeille sauvage de plus petite taille (11-13mm) spécialisée dans la récolte de pollen sur les Onobrychis (Müller et al. 2008).

Les chalicodomes se font de plus en plus rares en Europe occidentale. A titre d'exemple, Fabre (1882) citait $M$. (C.) sicula comme l'un des Hyménoptères les plus abondants au mois de mai dans le département de Vaucluse (France). Le déclin important des populations de $M$. (C.) parietina à l'échelle européenne est encore mal compris (Rasmont et al. 2003; Müller et al. 2006), même s'il existe un parallèle évident entre la régression des plantes-hôtes comme le sainfoin, la dégradation des sites naturels et l'apparente rareté de $M$. (C.) parietina en Europe occidentale, et particulièrement en France où il ne subsiste probablement que peu de populations isolées, dont quelques-unes ont été détectées en 2010 sur les communes de Fréjus (Var, obs. NJ Vereecken), du Mas-d'Azil (Ariège, obs. J Spéckens) et de La MureArgens (Alpes de Haute Provence, obs. M Aubert). La situation actuelle de $M$. (C.) sicula est difficile à évaluer, tant les données contemporaines sont rares en France où des prospections ciblées mériteraient d'être menées pour préciser sa distribution méditerranéenne.

\section{Références bibliographiques}

Berland L, 1934. Un cas probable de parthénogenèse géographique chez Leucospis gigas (Hyménoptère). Bulletin de la Société Zoologique de France 59: 172-175.

Bischoff H, 1927. Biologie der Hymenopteren. Julius Springer, Berlin.

Bosch J, 1992. Parasitism in wild and managed populations of the almond pollinator Osmia cornuta LATR. (Hymenoptera: Megachilidae). Journal of Apicultural Research 31: 77-82.

Bouček Z, 1974. A revision of the Leucospidae (Hymenoptera : Chalcidoidea) of the World. Bulletin of the British Museum (Nat. Hist.) Entomology 23: 1-241.
Bytinski-Salz H, 1963. Geographical variation and sex-ratio in Leucospis gigas (Hymenoptera, Chalcidoidea). Acta Entomologica Musei Nationalis Pragae 35: 527-530.

Ceballos G, 1956. Catalogo de los Himenopteros de España. C o n s e j o Superior de Investigaciones Cientificas - Instituto Español de Entomologia, Madrid.

Glausen CP, 1940. Entomophagous Insects. McGraw Hill, New York \& London.

Fabre J-H, 1882. Souvenirs entomologiques - Etudes sur l'instinct et les moeurs des insectes. (2 ${ }^{\text {ème }}$ Edition, $2^{\text {ème }}$ Série). Libraire Ch Delagrave, Paris.

Fabre J-H, 1886. Souvenirs entomologiques - Etudes sur l'instinct et les moeurs des insectes. ( $3^{\text {ème }}$ Edition, $3^{\text {ème }}$ Série). Libraire Ch Delagrave, Paris.

Habu A, 1962. Chalcididae, Leucospidae and Podagrionidae (Insecta: Hymenoptera). Dans Fauna Faponica, Biogeographical Society of Japan, Tokyo.

Jurine L, 1807. Nouvelle méthode de classer les Hyménoptères et les Diptères. JJ Paschoud, Genève.

Le Goff G, 2007. La nidification de Megachile (Chalicodoma) pyrenaica LePeletier dans la Province de Granada (Espagne) (Hymenoptera Apoidea Megachilidae). Lambillionea CVII 2: 277-281.

Linsenmaier W, 1959. Revision der Familie Chrysididae (Hymenoptera) mit besonderer Beriicksichtigung der europaischen Species. Mitteilungen der Schweizerischen Entomologischer Gesellschaft 32(1) : 1-232.

Müller A, 2010. Palaearctic Osmüne Bees. ETH Zürich, blog consulté le 10.x.2010 à l'adresse http://blogs.ethz.ch/osmiini

Müller A, Krebs A \& Amiet F, 1997. Bienen, mitteleuropäische Gattungen, Lebensweise, Beobachtung. Naturbuch Verlag, Augsburg.

Müller A, Diener S, Schnyder S, Stutz K, Sédivy G \& Dorn S, 2006. Quantitative pollen requirements of solitary bees: implications for bee conservation and the evolution of bee-flower relationships. Biological Conservation 130: 604-615.

Pagliano G, 1993. Catalogo degli imenotteri italiani. IV. (Apoidea: Colletidae, Andrenidae, Megachilidae, Anthophoridae, Apidae). Memorie della Società Entomologica Italiana 72: 331-467.

Pérez J, 1889. Les abeilles. Librairie Hachette et Cie, Paris.

Rasmont P, Barbier Y, Iserbyt S, Wahis R \& Terzo M, 2003. Jean-Henri Fabre pourrait-il observer aujourd'hui tous ces insectes? Actes du colloque International sur l'Entomologie, Jean-Henri Fabre, un autre regard sur l'insecte. CG Aveyron, Rodez, pp. 209-220.

Rebmann O, 1969. Zur Nestbauweise von Megachile (Chalicodoma) parietina (FOURCROY) (Hym., Apidae). Entomologische Zeitschrift 79(12): 138-139.

Tepedino V, 1988. Host discrimination in Monodontomerus obsoletus FABRICIUS (Hymenoptera: Torymidae), a parasite of the alfalfa leafcutting bee Megachile rotundata (FABRICIUS) (Megachilidae). Fournal of the New-York Entomological Society 96(1): 113-118.

Tkalcu B, 1977. Taxonomisches zu einigen Paläarktischen Bienenarten (Hymenoptera: Apoidea). Vestník Ceskoslovenské Spolecnosti Zoologické 41: 223-239.

Vincens N, Bosch J \& Blas M, 2009. Biology and population structure of Osmia tricornis LATREILLE (Hym., Megachilidae). Fournal of Applied Entomology 117: 300-306.

Westrich P, Schwenninger HR, Dathe HH, Riemann H, Saure G, Voith J \& Weber K, 1998. Rote Liste der Bienen (Hymenoptera: Apidae) Deutschlands. Dans Binot M, Bless R, Boye P, Gruttke H \& Pretscher P (Eds.), Rote Liste gefährdeter Tiere Deutschlands. Schriftenreihe Landschaftspflege Naturschutz 55: 119-129.

Westwood JO, 1835. On Leucospis, a genus of hymenopterous insects. Entomological Magazine 2: 212-218. 